\title{
Emerging trends in suicide prevention research
}

Nathalie Oexle ${ }^{1 *}$, Thomas Niederkrotenthaler ${ }^{2}$, Diego DeLeo $^{3}$

${ }^{1}$ Department for Psychiatry II, Ulm University and BKH Günzburg

${ }^{2}$ Unit Suicide Research \& Mental Health Promotion, Department of Social and Preventive

Medicine, Centre for Public Health, Medical University of Vienna

${ }^{3}$ Australian Institute for Suicide Research and Prevention, Griffith University

Word count: 2517 [max. 2500 words (excluding references)]

\section{Author of correspondence}

Address correspondence to Nathalie Oexle, Ulm University and BKH Günzburg, Department for Psychiatry II, Parkstraße 11, 89073 Ulm; phone: +49 731500 62304; email:

nathalie.oexle@uni-ulm.de 


\section{Abstract}

\section{Purpose of review}

The purpose of this article is to present notable findings and developments in suicide prevention research and in particular, strategies to detect persons at risk for suicide as well as strategies to reduce suicide risk among the general population and specific risk groups.

\section{Recent findings}

Popular strategies to detect persons at risk for suicide include scanning social media posts, prediction models using electronic health record data as well as the use of experienced sampling methodology to enhance our understanding of the suicidal process. Emerging approaches to reduce suicide risk include the development of media reporting recommendations, mass media campaigns to improve knowledge, attitudes and behaviour, as well as technology-enhanced interventions.

\section{Summary}

Recently, promising approaches to detect individuals at risk for suicide as well as effective strategies to reduce suicide risk emerged. Whether these new opportunities will translate into an effective reduction in suicide rates remains to be demonstrated.

\section{Keywords}

Suicide, Prevention, Suicide risk 


\section{Introduction}

Globally, around 800.000 people die by suicide every year [1]. Additionally, the World Health Organization (WHO) estimates that the number of suicide attempts can be at least 20 times higher [1]. Fatal or non-fatal suicide attempts generally leave family members, friends and other involved persons deeply affected, and result in significant costs for society [2]. In 2013, the WHO member states committed themselves to a significant reduction in suicide rates by 2020 [3]; however, until today suicide rates in many states have only slightly decreased and some countries like the US [4] even reported an increase in suicide rates in recent years. In general, effective suicide prevention requires: (i) strategies to detect individuals at risk, as well as (ii) strategies to reduce suicide risk among the general population and specific at-risk groups. This article aims to provide an overview of recent developments in research in both of these domains. We present and discuss those topics and studies we felt are most influential in driving current discussions and developments in suicide prevention research. As a review focusing on emerging trends in youth suicide prevention was recently published [5], we will focus on developments in adult suicide prevention.

Detecting persons at risk for suicide is difficult. Merely relying on self-report is errorprone, as suicidality is often concealed for fear of hospitalisation and other expected negative consequences [6]. Therefore, for some time now, besides their professional experience, clinicians evaluate suicide risk based on a person's membership within defined high-risk groups, such as those with history of suicide attempts, severe mood disorders or other psychiatric conditions, suicidal behaviour in the family and a variety of social factors. A multitude of such assumed risk factors exists; however, their accuracy in predicting suicide attempts was recently questioned. In their comprehensive systematic review, Franklin and colleagues [7] concluded that traditional suicide prevention research was not able to comprehensively identify causal risk factors for suicidal behaviour. Synthesizing results from 365 studies from the past 50 years, they found that the accuracy of known suicide risk factors 
in predicting suicide attempts is only slightly better than chance. Those findings emphasize limited knowledge regarding the validity of current suicide theories and an associated lack of approaches for early risk detection.

Suicide is a complex phenomenon and a multitude of suicide prevention strategies among the general population and specific risk groups exists. Despite large numbers of interventions aiming to reduce suicide, in 2016, a thorough systematic review reported that comprehensive evidence for effectiveness merely exists for restricting access to means of suicide, treatment of mental illnesses and some school-based prevention programs [8]. Many other strategies seem promising; however, the authors argued that methodologically sound studies are needed to judge their effectiveness in reducing suicide.

During the past two years, many interesting articles providing new evidence for established suicide prevention strategies as well as promising new approaches emerged. In the following paragraphs, we present notable approaches to (1) detect individuals at risk as well as (2) effective strategies to reduce suicide risk.

\section{(1) Detecting people at risk for suicide}

In recent years, new evidence regarding innovative and potentially effective approaches to detect persons at risk for suicide emerged, including scanning social media posts [9-16], prediction models using electronic health record data $[17,18]$, as well as the use of experienced sampling methodology to enhance our understanding of the suicidal process [1925]. We will present these three approaches and initial findings in the following paragraphs.

\section{Social media}

Concerning suicide prevention, the Internet and social media is a double-edged sword. For example, the Internet offers unlimited and unchecked information about suicide methods, whilst increased exposure to suicide-related content might also increases distress and suicide 
risk [26]. However, the freedom offered by the Internet also includes the possibility to anonymously share own suicidal ideation, and thereby reduces the threshold to express suicidal ideation and potentially address it with others. Scholars have argued that monitoring suicide-related social media posts could be used for detecting persons at risk for suicide and implementing early intervention; therefore this would help to prevent suicide [27]. Recently, researchers started to apply machine-learning technology to detect persons at risk for suicide based on their social media posting behaviour.

Indeed, a big part of modern social life happens online, and social media platforms such as Facebook and Twitter are increasingly used to express personal experiences of suicidality [9]. Initial evidence suggests that suicide-related messages on social media can indeed be an indicator for suicide risk. In the US and South Korea, suicide-related Twitter posts or web-blog entries were found to be associated with national suicide rates $[10,11]$. Over the past years, the algorithms to correctly detect high-risk messages on social media have continuously been improving [12-14]; however, conclusive evidence for a link between suicide-related posts on social media and actual suicide risk (particularly suicidal behaviour) is lacking. One study found that posting suicide-related messages on Twitter was linked to increased levels of suicidality based on a self-report measure [15], suggesting that suiciderelated social media posts can reflect actual suicide risk at the individual level. Bryan and colleagues reported that among deceased military personnel, social media posts of those who died by suicide vs. those who died by other causes differed both by content and pattern, suggesting that suicide risk might not only be indicated by the content of social media posts but also their patterns and contexts [16].

Despite these findings, the potential of this approach in improving the accuracy of suicide risk detection remains uncertain and more research on the link between suicide-related social media posts and actual suicide risk is needed. Scholars should also keep in mind that persons at high risk for suicide often do not seek help and conceal their suicidality due to 
stigma, lack of time, preference for self-help and fear of involuntary hospitalization $[6,28]$. Approaches that include systematic screening of social media posts and consequent (unwanted) support or even the risk of involuntary hospitalization of persons detected to be at risk for suicide bear the risk of decreasing online help-seeking and disclosure when negative consequences are feared.

\section{Electronic health record data}

The detection of factors predicting future suicidal behaviour is difficult as suicidality is a multifactorial phenomenon with a multitude of risk factors working together. In addition, suicide is a rare event and large sample sizes are required to test the complex models predicting its occurrence. Due to their size and richness in information, electronic health record data offer the unique possibility to investigate the predictive power of large numbers of potential risk factors simultaneously. In 2017, Barak-Corren and colleagues [17] analysed data from almost two millions patients derived from two large medical centres in Boston and found convincing evidence that electronic health record data can indeed be used to predict future suicide. Their developed model included a multitude of potential risk factors (demographics, diagnostic codes, laboratory results, medications) and correctly predicted about half of all suicides and suicide attempts occurring within 3-4 years after baseline. Those findings were confirmed by another study reporting similar results [18] and suggest that using electronic health record data to identify and support persons at risk for suicide could greatly contribute to preventing suicides. However, as existing studies did not include representative samples, the general validity of developed models needs further assessment. Furthermore, the potential of electronic health record data to detect persons at risk for suicide greatly depends on data registries actually including data about suicidal behaviour, which is often not the case. 


\section{Experienced sampling methodology}

The field of suicide prevention research is characterized by great diversity in theories that try to explain and predict suicidality. Each theory includes a set of suicide risk factors, which on their own cannot fully explain suicide [7], resulting in a growing list with low specificity. One reason for limited knowledge about the occurrence, course, correlates and predictors of suicidality is due to past research measuring suicidality at one single point in time only, covering long retrospective periods, and/or assessing exclusively suicidal thoughts rather than suicidal behaviour [29]. To improve suicide prevention, information about the short-term predictors for suicidal thoughts and behaviour, and the factors that predict the transition from suicidal thoughts to behaviour is needed.

One innovative approach with the potential to overcome some of these barriers and foster our understanding of the suicidal process is the use of experienced sampling methodology (ESM). ESM allows for real-time assessment of suicidality as well as its potential correlates and predictors. In ESM studies, suicidality and other factors of interest (e.g. affective states) are assessed briefly multiple times a day using smartphones or other wearable devices. Initial evidence suggests that ESM-based assessment increases the chance of persons correctly reporting their experiences with suicidality and does not increase or decrease suicidality among participants [19-21]. Existing ESM-studies showed that suicidal thoughts often occur in brief episodes with quick onset and changes even within hours [2224]. Additionally, one study reported that correlates, predictors of suicidality and predictors of change in suicidal thoughts need to be distinguished [22,25]. For example, it seems that hopelessness does correlate and predict suicidal thoughts a few hours later; however, it seems not to predict changes in suicidal thoughts.

ESM offers the novel possibility to provide information about the short-term correlates and predictors of suicidality and therefore improve our understanding of the suicidal process, detect persons at risk for suicide and foster the development of effective strategies for suicide 
prevention. However, existing studies using ESM mostly assessed suicidal thoughts rather than suicidal behaviour, had small sample sizes and followed participants over relatively short periods. If future studies address those limitations, ESM has great potential for generating knowledge needed for effective suicide prevention.

\section{(2) Interventions to reduce suicide risk}

Suicide is a complex phenomenon and its prevention requires a multitude of strategies working together [30]. Interventions to reduce suicide can be universal (i.e. target the general population), selective (i.e. target persons at risk) or indicated (i.e. target persons already experiencing suicidality). However, some interventions can be grouped within two or more of these domains. Below we present recent findings regarding the effectiveness of some popular strategies to prevent suicide.

\section{Media reporting recommendations}

It is well established that in line with the Werther effect, media reports about suicide can trigger imitational suicides (for a summary of past research see [31]). However, this is not always the case. During the past 10 years an increasing number of studies reported that responsible suicide-related media reports could not only avoid the harmful Werther effect, but also contribute to suicide prevention, what was termed the Papageno effect. In two early population-based ecological studies published in 2009 and 2010, Niederkrotenthaler and colleagues found that while sensationalist reports about suicide are associated with higher suicide rates, reports highlighting that coping with suicidal ideation is possible are associated with lower suicide rates $[32,33]$. A recent empirical study conducted in Germany reported that neutral language in media reports about suicide (e.g. using the term "suicide" rather than "Freitod/ free death") can effectively prevent attitudinal support for suicide among readers [34]. While earlier studies surprisingly reported that articles including interviews with suicide 
experts can lead to imitational suicides [33], new evidence supports the claim that this effect might be due to such articles often also including sensationalist content. Several randomized controlled trials (RCTs) found that stand-alone information about suicide prevention on websites and in newspapers had beneficial effects in terms of a reduction of suicidal ideation and an increase in suicide-prevention related knowledge [35,36]. These findings particularly applied to materials that featured experts and lay individuals speaking about personal stories of how to cope with suicidality.

In summary, available studies support the existence of the Papageno effect, but further research is necessary to identify which specific contents work best for various target populations and outcomes such as suicidality, stigma, or help-seeking. When reporting about suicide, journalists should adhere to the updated recommendations recently published by the WHO [37].

\section{Mass Media Campaigns}

With the ultimate goal of reducing suicide, a multitude of social media campaigns to improve suicide-related knowledge (e.g. suicide warning signs), attitudes (e.g. to reduce stigma) and behaviour (e.g. to encourage help-seeking) targeting various audiences exist. Two recent systematic reviews independently concluded that mass media campaigns are indeed an important component of effective suicide prevention [38,39]. However, included studies varied in quality and reported heterogeneous findings. While findings regarding improvements in knowledge or attitudinal outcomes were fairly consistent, the impact of mass media campaigns on behavioural outcomes (such as help-seeking or self-harm) were inconsistent [39]. A novel campaign has been recently described by King and colleagues [40], who investigated the effects of a media campaign targeting suicidality, gender roles and helpseeking among men. The results showed significant positive effects on help-seeking intentions. Despite promising initial evidence, more research testing the effect of mass media 
campaigns on behavioural outcomes as well as the effects of specific campaign components including content, duration and coverage is needed.

\section{Technology-enhanced interventions}

Scholars have argued that technology-enhanced approaches might contribute to suicide prevention due to their wide reach and simple accessibility [27,41]. While this might be the case, comprehensive evidence for the effectiveness of technology-enhanced approaches in reducing suicide risk is still lacking. While Internet-based cognitive behavioural therapy (CBT) seems to be as effective as traditional face-to-face CBT in reducing psychiatric distress in general [42], in contrast to traditional CBT, Internet-based CBT was not found effective in reducing suicidality [43]. A systematic review by Witt and colleagues reported some evidence for the effectiveness of online and phone-based applications for the self-management of suicidal ideation and self-harm in reducing suicidality [44]. However, the authors criticised the low methodological quality of existing studies and their potential biases. In addition, caution is warranted when implementing technology-enhanced interventions: in comparing the effectiveness of psychotherapy alone (i.e. treatment as usual) and psychotherapy plus access to a supportive smartphone app (i.e. LifeApp'tite) in reducing suicide risk, Toole and colleagues observed smaller decreases in suicide risk among participants with access to the smartphone app [45]. In summary, existing studies on the effectiveness of technologyenhanced interventions in reducing suicide risk were far from being conclusive. While some types of interventions might contribute to suicide prevention, others could even produce unintended harmful effects.

\section{Conclusion}

Big data and machine learning hold great promise for suicide prediction. First aid and emergency wards personnel would probably benefit from the availability of algorithms 
permitting quick evaluation of their clients' risk of suicide. Wearable technologies are currently used for checking heart and blood pressure, calories spent, and number of steps and their reliable indication on levels of anxiety and mood is expected; equally, suicidal ideation could be detected. Responsible media reports have great potential to contribute to suicide prevention by reducing suicidality and improving suicide-related knowledge and attitudes. Mass media campaigns could have similar effects, but more research is needed. Finally, some technology-enhanced approaches could effectively support people experiencing suicidality; however, unintended harmful side effects are possible. Whether the presented new opportunities will translate into an effective reduction in suicide rates remains to be demonstrated.

\section{Key points}

- Successful suicide prevention requires strategies to detect persons at risk as well as strategies to reduce suicide risk among the general population and specific risk groups.

- Popular approaches to detect individuals at risk for suicide include scanning social media posts, prediction models using electronic health records data and the use of experienced sampling methodology to enhance our understanding of the suicidal process.

- Media recommendations on how to report about suicide and mass media campaigns are promising approaches to reduce suicide risk among the general population and specific risk groups.

- Some technology-enhanced approaches could be an effective complement or even substitute for traditional support strategies; however, unintended harmful side effects are possible and need to be thoroughly investigated and addressed.

\section{Acknowledgements}


None.

\section{Financial support and sponsorship}

None.

\section{Conflicts of interest}

None. 


\section{References}

1. World Health Organization. Preventing suicide: A global imperative. Geneva, Switzerland: World Health Organization; 2014.

2. Shepard DS, Gurewich D, Lwin AK, et al. Suicide and Suicidal Attempts in the United States: Costs and Policy Implications. Suicide \& Life-Threatening Behavior 2016; $46: 352-62$.

3. World Health Organization. Mental Health Action Plan 2013-2020. Geneva, Switzerland: World Health Organization; 2013.

4. Centers for Disease Control and Prevention. Suicide rising across the US: More than a mental health concern. https://www.cdc.gov/vitalsigns/pdf/vs-0618-suicide-H.pdf.

5. King CA, Arango A, Ewell Foster C. Emerging trends in adolescent suicide prevention research. Current Opinion in Psychology 2018; 22:89-94.

* This review presents an overview of emerging approaches to prevent suicide among adolescents.

6. Blanchard M, Farber BA. "It is never okay to talk about suicide": Patients' reasons for concealing suicidal ideation in psychotherapy. Psychotherapy Research 2018:Published online.

7. Franklin JC, Ribeiro JD, Fox KR, et al. Risk factors for suicidal thoughts and behaviors: A meta-analysis of 50 years of research. Psychological Bulletin 2017; 143:187-232.\#

** This comprehensive meta-analysis summarized research from the past 50 years and concluded that traditional research was not able to detect the causal risk factors of suicidal thoughts and behaviour.

8. Zalsman G, Hawton K, Wasserman D, et al. Suicide prevention strategies revisited: 10year systematic review. The Lancet Psychiatry 2016; 3:646-59.

** This comprehensive systematic review summarizes evidence regarding the effectiveness of suicide prevention strategies from the past 10 years. The authors conclude that there is no comprehensive evidence for the effectiveness of most suicide prevention approaches.

9. Robinson J, Cox G, Bailey E, et al. Social media and suicide prevention: a systematic review. Early Intervention in Psychiatry 2016; 10:103-21.

10. Jashinsky J, Burton SH, Hanson CL, et al. Tracking suicide risk factors through Twitter in the US. Crisis 2014; 35:51-59.

* One of the first studies assessing the link between suicide-related social media posts and national suicide rates.

11. Won H-H, Myung W, Song G-Y, et al. Predicting national suicide numbers with social media data. PloS ONE 2013; 8:e61809.

* One of the first studies assessing the link between suicide-related social media posts and national suicide rates.

12. O'Dea B, Wan S, Batterham PJ, et al. Detecting suicidality on Twitter. Internet Interventions $2015 ; 2: 183-88$. 
13. Vioules MJ, Moulahi B, Aze J, et al. Detection of suicide-related posts in Twitter data streams. IBM J. Res. \& Dev. 2018; 62:7:1-7:12.

14. Aladağ AE, Muderrisoglu S, Akbas NB, et al. Detecting Suicidal Ideation on Forums: Proof-of-Concept Study. Journal of Medical Internet Research 2018; 20:e215.

15. Braithwaite SR, Giraud-Carrier C, West J, et al. Validating Machine Learning Algorithms for Twitter Data Against Established Measures of Suicidality. JMIR Mental Health 2016; 3:e21.

* This study is the first to assess the association between suicide-related social media posts and individual suicidality.

16. Bryan CJ, Butner JE, Sinclair S, et al. Predictors of Emerging Suicide Death Among Military Personnel on Social Media Networks. Suicide \& Life-Threatening Behavior 2018; 48:413-30.

17. Barak-Corren Y, Castro VM, Javitt S, et al. Predicting Suicidal Behavior From Longitudinal Electronic Health Records. The American Journal of Psychiatry 2017; 174:154-62.

* This study used a large data set derived from electronic health records and developed a model that correctly predicted half of all suicides occurring within the study period.

18. Simon GE, Johnson E, Lawrence JM, et al. Predicting Suicide Attempts and Suicide Deaths Following Outpatient Visits Using Electronic Health Records. The American Journal of Psychiatry 2018; 175:951-60.

19. Husky M, Olié E, Guillaume S, et al. Feasibility and validity of ecological momentary assessment in the investigation of suicide risk. Psychiatry Research 2014; 220:564-70.

20. Law MK, Furr RM, Arnold EM, et al. Does Assessing Suicidality Frequently and Repeatedly Cause Harm? A Randomized Control Study. Psychological Assessment 2015; 27:1171-81.

* This study demonstrated that frequent and repeated assessment of suicidality as used within Experienced Sampling Methods does not increase or decrease suicidality among respondents.

21. Torous J, Staples P, Shanahan M, et al. Utilizing a Personal Smartphone Custom App to Assess the Patient Health Questionnaire-9 (PHQ-9) Depressive Symptoms in Patients With Major Depressive Disorder. JMIR Mental Health 2015; 2:e8.

22. Kleiman EM, Turner BJ, Fedor S, et al. Examination of real-time fluctuations in suicidal ideation and its risk factors: Results from two ecological momentary assessment studies. Journal of Abnormal Psychology 2017; 126:726-38.

* This is one of the first studies to use Experienced Sampling Methodology to assess realtime fluctuations in suicidal ideation.

23. Hallensleben N, Spangenberg L, Forkmann T, et al. Investigating the Dynamics of Suicidal Ideation. Crisis 2018; 39:65-69.

24. Nock MK, Prinstein MJ, Sterba SK. Revealing the form and function of self-injurious thoughts and behaviors: A real-time ecological assessment study among adolescents and young adults. Journal of Abnormal Psychology 2009; 118:816-27. 
25. Kleiman EM, Nock MK. Real-time assessment of suicidal thoughts and behaviors. Current Opinion in Psychology 2018; 22:33-37.

** This review provides a comprehensive overview on the potential of using experienced sampling Methodology to assess suicidal thoughts and behaviour.

26. Thornton L, Handley T, Kay-Lambkin F, et al. Is A Person Thinking About Suicide Likely to Find Help on the Internet? An Evaluation of Google Search Results. Suicide \& Life-Threatening Behavior 2017; 47:48-53.

27. Notredame C-E, Grandgenèvre P, Pauwels N, et al. Leveraging the Web and Social Media to Promote Access to Care Among Suicidal Individuals. Frontiers in Psychology 2018; 9.

28. Czyz EK, Horwitz AG, Eisenberg D, et al. Self-reported Barriers to Professional Help Seeking Among College Students at Elevated Risk for Suicide. Journal of American College Health 2013; 61:398-406.

29. Glenn CR, Nock MK. Improving the short-term prediction of suicidal behavior. American Journal of Preventive Medicine 2014; 47:S176-80.

30. Hawton K, Pirkis J. Suicide is a complex problem that requires a range of prevention initiatives and methods of evaluation. The British Journal of Psychiatry 2017; 210:381-83.

31. Niederkrotenthaler T, Stack S. Media and suicide: International perspectives on research, theory, and policy / Thomas Niederkrotenthaler and Steven Stack, editors. New Brunswick: Transaction Publishers; 2017.

* Book that provides a comprehensive overview of the link between media reports and suicide prevention.

32. Niederkrotenthaler T, Till B, Kapusta ND, et al. Copycat effects after media reports on suicide: a population-based ecologic study. Social Science \& Medicine 2009; 69:1085-90.

33. Niederkrotenthaler T, Voracek M, Herberth A, et al. Role of media reports in completed and prevented suicide: Werther v. Papageno effects. The British Journal of Psychiatry $2010 ; 197: 234-43$.

* First study providing evidence for the Papageno effect.

34. Arendt F, Scherr S, Niederkrotenthaler T, et al. The role of language in suicide reporting: Investigating the influence of problematic suicide referents. Social Science \& Medicine 2018; 208:165-71.

35. Till B, Arendt F, Scherr S, et al. Effect of Educative Suicide Prevention News Articles Featuring Experts With vs Without Personal Experience of Suicidal Ideation: A Randomized Controlled Trial of the Papageno Effect. The Journal of clinical psychiatry $2018 ; 80$.

36. Till B, Tran US, Voracek M, et al. Beneficial and harmful effects of educative suicide prevention websites: randomised controlled trial exploring Papageno v. Werther effects. The British Journal of Psychiatry 2017; 211:109-15.

37. World Health Organization. Preventing Suicide. A Resource for Media Professionals: Update 2017. Geneva, Switzerland: WHO; 2017.

38. Torok M, Calear A, Shand F, et al. A Systematic Review of Mass Media Campaigns for Suicide Prevention: Understanding Their Efficacy and the Mechanisms Needed for 
Successful Behavioral and Literacy Change. Suicide \& Life-Threatening Behavior 2017; 47:672-87.

* This systematic review provides a comprehensive overview of existing evidence regarding the role of mass media campaigns in suicide prevention.

39. Pirkis J, Rossetto A, Nicholas A, et al. Suicide Prevention Media Campaigns: A Systematic Literature Review. Health Communication 2017:1-13.

40. King KE, Schlichthorst M, Spittal MJ, et al. Can a documentary increase help-seeking intentions in men? A randomised controlled trial. Journal of Epidemiology and Community Health 2018; 72:92-98.

41. Kreuze E, Jenkins C, Gregoski M, et al. Technology-enhanced suicide prevention interventions: A systematic review. Journal of Telemedicine and Telecare 2017; 23:60517.

* This systematic review summarizes existing technology-enhanced suicide prevention approaches and their effectiveness.

42. Carlbring P, Andersson G, Cuijpers $P$, et al. Internet-based vs. face-to-face cognitive behavior therapy for psychiatric and somatic disorders: an updated systematic review and meta-analysis. Cognitive Behaviour Therapy 2018; 47:1-18.

43. Leavey K, Hawkins R. Is cognitive behavioural therapy effective in reducing suicidal ideation and behaviour when delivered face-to-face or via e-health? A systematic review and meta-analysis. Cognitive Behaviour Therapy 2017; 46:353-74.

44. Witt K, Spittal MJ, Carter G, et al. Effectiveness of online and mobile telephone applications ('apps') for the self-management of suicidal ideation and self-harm: a systematic review and meta-analysis. BMC Psychiatry 2017; 17:297.

45. O'Toole MS, Arendt MB, Pedersen CM. Testing an App-Assisted Treatment for Suicide Prevention in a Randomized Controlled Trial: Effects on Suicide Risk and Depression. Behavior Therapy 2018.

*This study revealed that some technology-enhanced approaches to prevent suicides might have unintended harmful effects. 